\title{
Therapy of cancer by cytokines mediated by gene therapy approach
}

\author{
Cheng Qian ${ }^{1,3}$, Xin Yuan Liu ${ }^{1,2}$, Jesus Prieto ${ }^{3}$ \\ ${ }^{1}$ Xinyuan Institute of Medicine and Biotechnology, School of Life Sciences, Zhejiang Sci-Tech University, Hangzhou, China; ${ }^{2}$ Institute \\ of Biochemistry and Cell Biology, Chinese Academy of Sciences, Shanghai 200031, China; ${ }^{3}$ Division of Gene Therapy and Hepatol- \\ ogy, CIMA, University of Navarra, Pamplona 31008, Spain
}

Gene therapy offers a new approach for treatment of cancer. Transfer of genes encoding immunostimulatory cytokines has been used with remarkable success to eliminate cancer in animals. However, clinical trials in patients with this strategy had limited efficacy. Therefore, improvement of gene transfer vector system is necessary. A hybrid viral vector, consisting of SFV replicon with either murine IL-12 or reporter LacZ gene, was constructed. This hybrid vector showed specificity and high level of expression in HCC both in vitro and in vivo. In a rat orthotropic liver tumor model, treatment of established tumors by the hybrid vector with mIL-12 gene resulted in a strong anti-tumor activity without accompanying toxicity. Subsequently, a helper-dependent adenovirus vectors containing a mifepristone (RU486) inducible system was constructed for controlled and liver-specific expression of human interleukin 12 (hIL-12) (HD-Ad/RUhIL-12) and mouse IL-12 (mIL-12) (HD-Ad/RUmIL-12). Data showed that high and sustained serum levels of hIL-12 could be attained by continuing administration of RU486 every 12 or $24 \mathrm{~h}$. Repetitive induction of hIL-12 could be obtained over, at least, a period of 48 weeks after a single injection of HD-Ad/RUhIL-12. Treatment of liver metastases with of HD-Ad/RUmIL12 plus RU846 resulted in complete tumor regression in all animals. Then, different cytokine genes were inserted into conditional replicative adenoviruses vectors (also called oncolytic adenovirus). Replication of adenovirus in tumor cells would kill tumor cells and release viruses, which infect surrounding tumor cells. The combination of cytopathic effect by oncolytic adenovirus and biological effect of transgene would exert strong antitumor activity. These new types of vectors may provide a potent and safe tool for cancer gene therapy.

Cell Research (2006) 16:182-188. doi:10.1038/sj.cr.7310025; published online 13 February 2006

Keywords: gene therapy, cancer, cytokines, vectors, adenovirus

\section{Introduction}

Malignant cells in tumors harbor mutated or overexpressed proteins that can be recognized by cell-destroying mechanisms of the immune system. Tumors that can evade anti-tumoral immunity due to poor antigenicity of tumoral antigens or to the lack of expression of MHC molecules by the transformed cells and/or to the secretion of immunosuppressive factors (such as TGF-beta or VEGF) [1,2].

Cytokines play important roles in inflammatory and immune responses. Some have important effects on tumor growth by augmenting anti-tumor immune responses,

Correspondence: Cheng Qian

Tel: 34-948-194700; Fax: 34-948-194717;

E-mail: cqian@unav.es inducing apoptosis in cancer cells or impairing the formation of new tumoral vessels. In the last two decades, several cytokines have been shown to exert a relevant antitumor activity both in experimental models and human. However, the clinical application has been compromised by side effects following systemic delivery of the recombinant protein. Hence, the rationale for cytokine-based gene therapy is that local expression of these molecules may result in enhanced anti-tumor activity and reduced toxicity [3-5].

\section{Gene transfer of cytokines by replication-defective adevnoviral vector}

IL-12 may be the most potent anti-tumoral cytokine. It can induce a TH1 type of response, activate NK cells and cytotoxic T lymphocytes, inhibit tumoral neoangiogenesis, 
and enhance the expression of adhesion molecules on endothelial cells, facilitating the traffic of lymphocytes to the tumor [6]. IL-12, however, is toxic when administered systemically as a recombinant protein [6]. The rational for IL-12 gene therapy is to allow local production of the cytokine at the tumor site thus achieving high intratumour or peritumoral levels with low serum concentration [3-6]. This procedure would therefore maximize the anti-tumoral effect of the cytokine while minimizing its systemic toxicity.

It has been shown that intratumoral administration of a recombinant adenovirus encoding IL-12 (AdIL-12) to animals with different types of carcinoma caused complete tumor eradication in most of animals and increased longterm survival [7-9]. More importantly, it was well reported in animal models with orthotropic tumors that more closely resembles human diseases such as hepatocellular carcinoma [9], gliomas [10], prostate carcinomas [11], medullary thyroid carcinoma [12], and liver metastases from colorectal [7] or breast cancer [13]. In most cases, animals that showed complete tumor regression were resistant against tumor rechallenge. Moreover, injection of AdIL-12 in one nodule of tumor resulted in regression of the distant nodule of tumor [9]. This effect has been attributed to the fact that a proportion of the adenovirus injected intratumorally escapes to the general circulation and, due to the intense hepatotropism of adenoviruses, the circulating virions infect the hepatic tissue surrounding the tumor nodules [14]. The IL-12 produced in tumor and by hepatocytes adjacent the neoplastic nodules may induce strong specific anti-tumor immunity via activating NK cells and enhancing the expression of adhesion molecules in the tumor, and subsequently result in powerful anti-angiogenic effect and tumor regression [9, 14]. AdIL-12 administrated via intra-hepatic arterial route was also shown to be efficient in the treatment of a very aggressive model of multifocal hepatocellular carcinoma in rats, causing a significant reduction of tumor burden and prolongation of survival [9].

IL-12 gene transfer to tumor cells also works in cancer arising spontaneously in large animals such as gray horses, that develop metastatic melanoma [15] or woodchucks [16]. The latter provide a model of hepatocellular carcinoma that very closely resembles the one that complicates human HBV-related liver cirrhosis. Woodchucks infected with the WHV develop chronic liver disease and multinodular tumors. Intratumoral injection of adenovirus vectors encoding for murine interleukin 12 and the costimulatory molecule B7.1 into large $(2-5 \mathrm{~cm})$ liver tumor nodules resulted in substantial tumor regression [16].

As it has been indicated, clinical development of recombinant IL-12 for the treatment of cancer was hindered by unexpected toxicities in an early clinical trial due to what we now know as down-regulation of the pharmacokinetic/ pharmacodynamic response. However, recombinant IL-12 can be given subcutaneously and partial tumor regressions have been observed in melanoma and renal cell carcinoma, main toxicity including elevated liver function tests and leukopenia [17], mild nausea and fatigue [18].

Regarding gene therapy, clinical trials have been carried to evaluate the feasibility and safety of intratumoral injection of an adenoviral vector encoding human IL-12 genes for advanced tumors in the digestive system [19]. The vector encoding for IL-12 was injected intratumorally in doses ranging from $2.5 \times 10^{10}$ to $3 \times 10^{12}$ viral particles to seven cohorts of patients with advanced pancreatic, colorectal, or primary liver malignancies. Patients with stable condition and no other serious adverse reactions were allowed to receive up to 3 monthly doses. The 21 patients received a total of 44 intratumoral injections. Therapy was well tolerated, and dose-limiting toxicity was not observed. Frequent but transient adverse reactions, including fever, malaise, sweating, and lymphopenia, seemed to be related to vector injection rather than the transgene expression, and no cumulative toxicity was observed. In four of 10 assessable patients, a significant increase in recruitment of immune cells was apparent in the tumor sites. Three tumor regressions ( 2 minor and 1 partial) of the injected tumor masses were observed among patients with hepatocellular carcinoma. Stable condition was observed in $29 \%$ of patients, most of these suffered from primary liver cancer. However, we were not able to detect any sign of systemic activity since no tumor remissions were observed in non-injected tumor sites and no shift was observed in the delayed hypersensitivity skin tests using autologous tumor extracts.

Despite the results from this early trial were not extremely impressive, it is very important and probably is proof-of-concept that local production of IL-12 through vector injection inside a tumor can enhance the recruitment of lymphocytes into tumor infiltration, and in some cases accompanied with tumor regression. In order to achieve a stronger effect for routine clinical application, potent gene transfer vectors, which provide more intensive or more sustained local production of cytokines, should be developed.

\section{Improvement of gene transfer vectors}

\section{Hybrid vector for gene therapy of cancer}

The vectors, capable of generating high levels of transgenic proteins in the tumor and enhancing more intense antitumor responses, should be developed [20]. However, high-expression vectors may increase the risk of toxicity. Thus, avoidance of transgene expression at extratumoral 
sites should be absolute necessary for the use of such gene therapy tools [20]. Targeting of tumor specific promoters at transcriptional level is another strategy [21]. However, these are normally weak promoters that do not allow high level expression of the transgenic protein in the neoplastic tissue $[21,22]$. Thus, it is necessary for the treatment of cancer to achieve both transcriptional targeting and high level expression of the transgene. Furthermore, when the transgene is an immunostimulatory cytokine, the anti-tumor effect will be enhanced if the transgenic expression is able to induce apoptosis in the neoplastic cells. It has been shown that the release of apoptotic bodies in a cytokinerich milieu would favor the presentation of tumor antigens and the induction of anti-tumor immunity [23]. Therefore, four criteria is established for constructing of a successful vector for the gene therapy of cancer: high infectivity for tumor cells; specific for tumor targeting; high production of the therapeutic protein inside the tumor and induction of selective apoptosis of tumor cells.

Alphaviruses are RNA enveloped viruses that contain a single positive strand RNA molecule as genome [24, 25]. Different alphaviruses such as Sindbis virus, Semliki Forest virus (SFV), and Venezuelan Equine Encephalytis virus have served for the construction of gene therapy vectors. These are based on the use of self-replicating RNA molecules derived from alphavirus genomes in which the 5'and 3' sequences necessary for replication and the replicase (Rep) gene have been maintained, while the genes coding for the viral structural proteins have been deleted and substituted by a transgene. Upon transfecting the vector into a cell, Rep will be translated and copy the vector RNA into a negative strand RNA that will be used as template for the amplification of the vector RNA. Rep can also recognize a subgenomic promoter in the negative strand RNA from which it will make a smaller subgenomic RNA that can be translated to produce the heterologous protein.

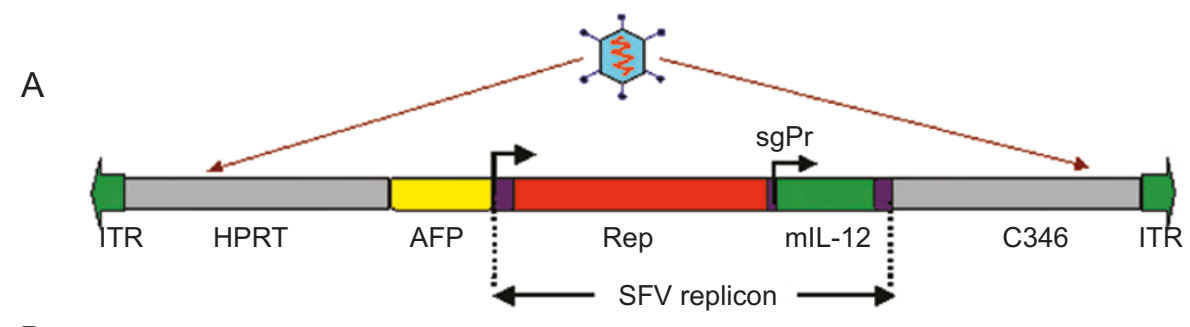

B
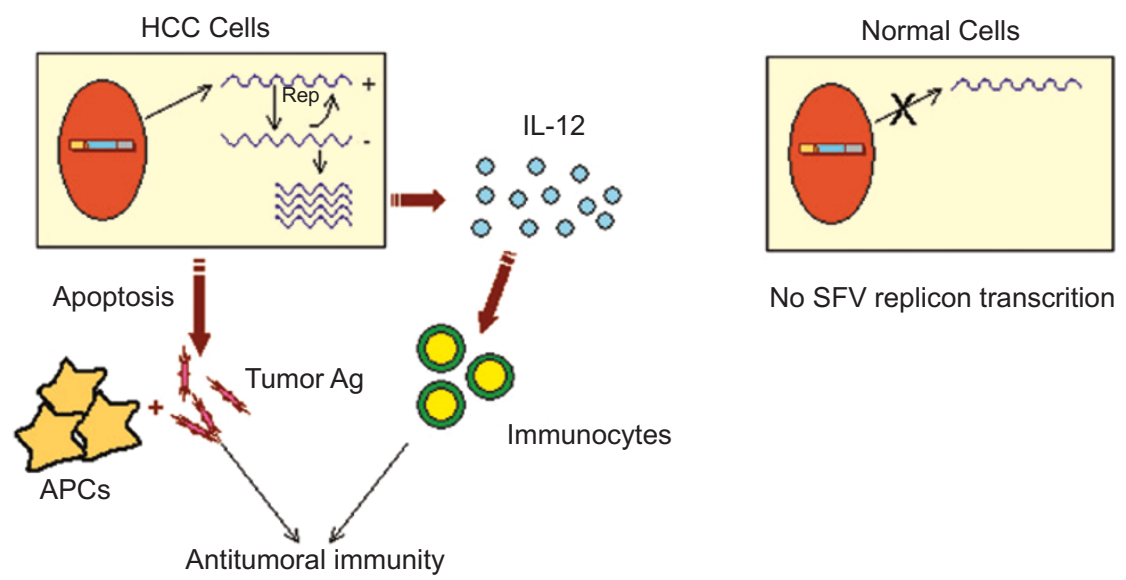

No SFV replicon transcrition

Figure 1 Diagram of gutless adenovirus-SFV hybrid vector and its potential antitumoral activity. (A) The hybrid vector contains a gutless adenovirus sequence in which a SFV replicon has been inserted under the control of the AFP promoter/enhancer (AFP). The heterologous gene mIL-12 is placed under the control of the SFV subgenomic promoter ( $\mathrm{sg}$ Pr). (B) Upon infection of HCC cells (left) with the hybrid vector the SFV replicon mRNA can be transcribed from the tumor-specific promoter, Rep will be translated and copy SFV RNA genome (+) into negative strand RNA (-). Rep will also synthesize a SFV subgenomic RNA from which mIL-12 will be expressed at high levels. The mIL-12 secreted from infected cells will activate immunocytes at the site of infection. In addition, SFV replication will also induce apoptosis in infected cells leading to the release of tumor antigens from apoptotic cells, which can be taken up by antigen presenting cells (APCs). Presentation of tumor antigen by APCs and activation of immunocytes by IL-12 will activate immune responses against the tumor. However, if this hybrid vector infects normal cells (right), the SFV replicon mRNA will not be transcribed and therefore, neither transgene expression nor apoptosis will occur. 
Replication and expression of the transgene occur at very high levels and is associated with the induction of apoptosis in the infected cells $[25,26]$.

A hybrid adenoviral vector has been constructed that contains the sequence of a recombinant SFV replicon under the transcriptional control of AFP promoter (Figure 1). In this construct the SFV replicon contained the IL-12 gene driven by the SFV subgenomic promoter. Our aim was to achieve specific induced apoptosis in AFP-expressing HCC tumors but not in other, high level of replication of vector with IL-12, and consequently to gain the treatment of liver cancer with safety and efficacy.

Our results showed that hybrid vectors induced both specific and high level expression of transgenes in AFPexpressing HCC cells, but not in non-HCC cells. HCC cells infected with hybrid vectors were selectively eliminated due to the induction of apoptosis by SFV replication. The specificity of this vector was confirmed in vivo that a high level of lacZ reporter gene expression was only observed in tumors of those animals. In addition, a high percentage of tumor cell apoptosis was induced in those tumors. No transgene expression or cell apoptosis was detected in normal liver following administration of the hybrid vector. In a rat orthotropic liver tumor model, treatment of established tumors by the hybrid vector carrying mIL-12 gene resulted in a strong anti-tumoral activity with substantial tumor regression. Tumor-free long-term survival was found in $50 \%$ of treated animals. No systemic or liver toxicities were observed. Our data suggest that this new type of adenovirus-alphavirus hybrid vector may provide a potent and safe tool for cancer gene therapy [27].

\section{Oncolytic viruses for gene therapy of cancer}

Virotherapy with oncolytic viruses is based on the use of tumor specific replicating virus. In this approach, transduced cells are eliminated as a consequence of virus infection and replication. Moreover, these infected cells can be the resources for producing new infectious virus particles that will infect surrounding tumor cells and spread throughout the tumor. Adenovirus has been genetic modified as tumor selective replicating virus. The first approach of this vector that has been developed, named mutant $d l 1520$ or ONYX-015, was based on deletion of the E1B 55K gene of adenovirus. Heise et al demonstrated that ONYX-015 infected and replicated efficiently within tumors in model of metastatic liver tumor following i.v. administration. Viral replication within tumors was associated with tumor regressions [28]. Another approach to restrict adenovirus replication in tumor cells is based on selective expression of E1 gene in tumor cells mediated by tumor specific transcriptional elements. The alpha-fetoprotein (AFP), hTERT and E2F promoters have been applied. A hepatocellular carcinoma specific adenorirus CV890 has been constructed based on use of AFP promoter [29]. It was reported that CV890 induced cytotoxicity and progeny virus production only in HCC tumor with AFP production. A significant inhibition of tumor growth was observed in nude mice bearing human Hep3B HCC tumors [29]. Similarly, strong anti-tumor effect was observed in different HCC tumor models by oncolytic adenoviral vector based on use of hTERT and E2F promoters [30-32].

Takahashi et al combined these two replicating strategies to engineer adenovirus more selective for tumor therapy. In their study, they constructed a recombinant replication-competent adenovirus (rRCAd; AdAFPep/Rep) that expresses both E1A-13S driven by the alpha-fetoprotein (AFP) enhancer/promoter (AFPep) lacking any silencers in the 5'-flanking region of the AFP gene, and 55K-deleted E1B driven by the cytomegalovirus (CMV) promoter. They demonstrated that rRCAd virus can lyse AFP-producing HCC cells with a higher specificity and potency than previously reported. Furthermore, this virus was capable of complete eradication of a preestablished Huh-7 tumor by a single intratumoral injection of $10^{8}$ plaque-forming units (pfu) of AdAFPep/Re [33]. Kim et al used a same strategy to construct another adenovirus YKL-1001. It induced both cytopathic effects exclusively in AFP-positive liver cancer cells and the growth inhibition of pre-established Hep3B xenografts [34].

The oncolytic viruses not only have capacity for destruction of tumors but also can be used as potential vectors to express therapeutic genes in tumor cells. Bristol et al. has used oncolytic adenoviruses to express granulocyte-macrophage colony-stimulating factor (GM-CSF). They found that oncolytic adenovirus with GM-CSF elicited greater efficacy compared to oncolytic adenovirus in an animal model with HCC tumor [35]. Genes coding for inducer of apoptosis such as tumor necrosis factor-related apoptosisinducing ligand (TRAIL), second mitochondria-derived activator of caspases (Smac), and Mda7/IL-24 have been incorporated into oncolytic adenovirus. The tumor regression was observed in animal models with established tumor after treatment with these vectors [36-38].

\section{Long-term expression vectors for gene therapy of cancer}

Another reason for the lack of efficacy likely depends on short duration of transgene expression and on the fact that the tumor tissue is not easily infected with adenoviral vectors. Several preclinical data suggest that sustained and long-term expression of therapeutic genes may increase the efficacy against cancer. Thus in order to be effective, it would be necessary to infect peritumoral tissue with long term expression vectors encoding cytokines endowed with potent immunostimulant and antiangiogenic properties 
[39]. However, these potent biological molecules may be potentially toxic $[32,33]$, if they are expressed in a sustained manner. Hence, when using long-term expression vectors for transfer of anti-tumor cytokines it would be essential to strictly control their expression in order to reach therapeutic levels while avoiding unwanted side effects.

The ability to regulate gene expression by using small molecules as ligands has been explored previously [40]. Four major systems have been developed, including regulation by the antibiotic tetracycline (Tet), the insect steroid ecdysone or its analogs, the antiprogestin mifepristone (RU486), and chemical "dimerizers" such as the immunosuppressant rapamycin and it analogs [40-42]. They all involve the drug-dependent recruitment of a transcriptional activation domain to a basal promoter driving the gene of interest, but differ in the mechanism of recruitment. In the mifepristone system, drug-regulated transcription is achieved by fusing a heterologous DNA binding domain (DBD) of yeast GAL4 protein and activation domain (AD) of VP-16 or NF-kB p65 proteins to a mutant human progesterone receptor that is unaffected by endogenous hormones but is activated by synthetic antiprogestins, at doses sufficiently low to avoid side-effects in human [43]. The properties of the mifepristone-regulated system have been investigated in transgenic animals and naked DNA plasmid in muscles [44]. Burcin et al [43] have incorporated this system into a gutless adenovirus with a human growth hormone (hGH) target gene. They showed that background transcription was undetectable in vitro and in vivo and that, over 50 days, hGH production could be cycled on and off three times, or maintained at steady state levels, by delivery of the inducer.

Recently we generated a gutless adenovirus vector encoding human IL-12 (hIL-12) and murine IL-12 (mIL-12) with a liver-specific mifepristone-regulable system. The data showed that infection of cells with HD-Ad/RUhIL-12 resulted in high level of hIL-12 expression in the presence of RU486 only in hepatocytes. In animals injected with HD-Ad/RUhIL-12 the administration of RU486 induced a transient rise of serum hIL-12 that peaked at $10 \mathrm{~h}$ and completely disappeared by $72 \mathrm{~h}$. The peak value of hIL-12 was dependent on the doses of the vector and the inducer. High and sustained serum levels of hIL-12 could be attained by continuing administration of RU486 every 12 or $24 \mathrm{~h}$. Repetitive induction of hIL-12 could be obtained over, at least, a period of 48 weeks after a single injection of HD-Ad/RUhIL-12. Although the vector was detected in many tissues after systemic injection, transcription of the transgene was limited in the liver only. Treatment of liver metastases with $5 \times 10^{8}$ iu of HD-Ad/RUmIL-12 plus RU486 resulted in complete tumor regression in all animals. Gutless adenoviral vectors allow liver-specific and regulable transgene expression for prolonged periods of time. These vectors are promising tools for gene therapy of liver cancer and could also be useful for other forms of hepatic disease [45].

\section{Final remarks}

Gene therapy has emerged as a powerful tool to regulate biological functions in diseased tissues with application in practically all medical fields. An increasing number of experimental and clinical studies clearly underline the important role of genes to serve as a curative drug in future. However, intensive researches are still required to evaluate the potential of gene therapy, to improve the efficacy and minimize the toxicity of the procedure. Future efforts should be directed to: 1) the development of vectors with high transduction efficiency, high transgene capacity, and acceptable toxicity profile, 2) development of systems allowing desired duration and regulation of the gene expression, 3) identification of the ideal therapeutic gene or gene combinations for each therapeutic indication, and 4) development of innovative methods for large-scale industrial production to allow accessible prices for wide access to these new drugs. Intensive testing in animal models to ensure drug safety and functionality is needed before contemplating clinical applications. Furthermore, clinical trials designed carefully under strict regulatory conditions, could provide more useful information on the potential of gene therapy in humans, especially in devastating diseases without effective therapy, as in cases of non-resectable and chemo-resistant tumors. In addition, with new imaging techniques such as Positron Emission Tomography (PET) it can potentially detect and quantitate the function of the transferred gene in vivo, which could be a powerful tool for monitoring the progress of clinical gene therapy [46].

\section{Acknowledgements}

This work was supported by the Tech Research and Development Program of China(" 863 " program, No. 2002AA216021), National Basic Research Program of China (“973” program, No. 2004CB518804), grant QLK31999-00364 from EU, UTE project of CIMA and grant C03/02 from Instituto Carlos III, Spain.

\section{References}

1 Roth C, Rochlitz C, Kourilsky P. Immune response against tumors. Adv Immunol 1994; 57:281-351.

2 Gabrilovich DI, Chen HL, Girgis KR, et al. Production of vascular endothelial growth factor by human tumors inhibits the functional maturation of dendritic cells. Nature Med 1996; 
2:1096-103.

3 Melero I, Mazzolini G, Narvaiza I, et al. IL-12 gene therapy for cancer: in synergy with other immunotherapies. Trends Immunol 2001; 22:113-5.

4 Qian C, Sangro B, Prieto J. New strategies to enhance gene therapy efficiency. Gastroenterology 2002; 123: 639-42 .

5 Prieto J, Qian C, Sangro B, Melero I, Mazzolini G. Biological therapy of liver tumors. Surg Clin North Am 2004; 84:643-57.

6 Trinchieri G. Interleukin-12: a cytokine at the interface of inflammation and immunity. Adv Immunol 1998; 70:83-243.

7 Caruso M, Pham-Nguyen K, Kwong YL, et al. Adenovirus-mediated interleukin-12 gene therapy for metastatic colon carcinoma. Proc Natl Acad Sci U S A 1996; 93:11302-6.

8 Mazzolini G, Qian C, Xie X, et al. Tumor regression and longterm protection for colon cancer after intratumoral injection of adenovirus expressing IL-12. Cancer Gene Ther 1999; 5:51422.

9 Barajas M, Mazzolini G, Genové G, et al. Gene Therapy of Orthotopic Hepatocellular Carcinoma in Rats Using Adenovirus Coding for interleukin-12 (IL-12). Hepatology 2001; 33:5261.

10 Liu Y, Ehtesham M, Samoto K, et al. In situ adenoviral interleukin 12 gene transfer confers potent and long-lasting cytotoxic immunity in glioma. Cancer Gene Ther 2002; 9:9-15.

11 Nasu Y, Bangma CH, Hull GW, et al. Adenovirus-mediated interleukin-12 gene therapy for prostate cancer: suppression of orthotopic tumor growth and pre-established lung metastases in an orthotopic model. Gene Ther 1999; 6:338-49.

12 Zhang R, DeGroot LJ. Gene therapy of a rat follicular thyroid carcinoma model with adenoviral vectors transducing murine interleukin-12. Endocrinology 2003; 144:1393-8.

13 Divino CM, Chen SH, Yang W, et al. Antitumor immunity induced by interleukin-12 gene therapy in a metastatic model of breast cancer is mediated by natural killer cells. Breast Cancer Res Treat 2000; 60:129-34.

14 Mazzolini G, Narvaiza I, Bustos M, et al. Alpha(v)beta(3) integrin-mediated adenoviral transfer of interleukin-12 at the periphery of hepatic colon cancer metastases induces VCAM-1 expression and T-cell recruitment. Mol Ther 2001; 3:665-72.

15 Heinzerling LM, Feige K, Rieder S, et al. Tumor regression induced by intratumoral injection of DNA coding for human interleukin 12 into melanoma metastases in gray horses. J Mol Med 2001; 78:692-702.

16 Putzer BM, Stiewe T, Rodicker F, et al. Large nontransplanted hepatocellular carcinoma in woodchucks: treatment with adenovirus-mediated delivery of interleukin 12/B7.1 genes. J Natl Cancer Inst 2001; 93:472-9.

17 Alatrash G, Hutson TE, Molto L, et al. Clinical and immunologic effects of subcutaneously administered interleukin-12 and interferon alfa-2b: phase I trial of patients with metastatic renal cell carcinoma or malignant melanoma. J Clin Oncol 2004; 22:2891-900.

18 Parihar R, Nadella P, Lewis A, et al. A phase I study of interleukin 12 with trastuzumab in patients with human epidermal growth factor receptor-2-overexpressing malignancies: analysis of sustained interferon gamma production in a subset of patients. Clin Cancer Res 2004; 10:5027-37.

19 Sangro B, Mazzolini G, Ruiz J, et al. Phase I trial of intratumoral injection of an adenovirus encoding interleukin-12 for advanced digestive tumors. J Clin Oncol 2004; 22:1389-97.

20 McCormick F. Cancer gene therapy: fringe or cutting edge? Nat Rev Cancer 2001;1:130-41.

21 Nettelbeck DM, Jerome V, Muller R. Gene therapy: designer promoters for tumor targeting. Trends Genet 2000;16:174-81.

22 Takikawa H, Mafune K, Hamada H, et al. An advanced strategy of enhanced specific gene expression for hepatocellular carcinoma. Int J Oncol 2003; 22:1051-6.

23 Tirapu I, Rodriguez-Calvillo M, Qian C, et al. Cytokine gene transfer into dendritic cells for cancer treatment. Curr Gene Ther 2002; 2:79-89.

24 Glasgow GM, McGee MM, Tarbatt CJ, et al. The Semliki Forest virus vector induces p53-independent apoptosis. J Gen Virol 1998; 79:2405-10.

25 Berglund P, Smerdou C, Fleeton MN, Tubulekas I, Liljeström P. Enhancing immune responses using suicidal DNA vaccines. Nat Biotech 1998; 16:562-5.

26 Rodriguez-Madoz JR, Prieto J, Smerdou C. Semliki forest virus vectors engineered to express higher IL-12 levels induce efficient elimination of murine colon adenocarcinomas. Mol Ther 2005; 12:153-3.

27 Guan M, Rodriguez-Madoz JR, Alzuguren P, et al. Increased efficacy and safety in the treatment of experimental liver cancer with a novel adenovirus-alphavirus hybrid vector. Cancer Res (In press).

28 Heise CC, Williams AM, Xue S, Propst M, Kirn DH. Intravenous administration of ONYX-015, a selectively replicating adenovirus, induces antitumoral efficacy. Cancer Res 1999; 59:2623-8.

29 Li Y, Yu DC, Chen Y, et al. A hepatocellular carcinoma-specific adenovirus variant, CV890, eliminates distant human liver tumors in combination with doxorubicin. Cancer Res 2001; 61:642836.

30 Zou W, Luo C, Zhang Z, et al. A novel oncolytic adenovirus targeting to telomerase activity in tumor cells with potent. Oncogene 2004; 23:457-64.

31 Jakubczak JL, Ryan P, Gorziglia M, et al. An oncolytic adenovirus selective for retinoblastoma tumor suppressor protein pathwaydefective tumors: dependence on E1A, the E2F-1 promoter, and viral replication for selectivity and efficacy. Cancer Res 2003; 63:1490-9.

32 Bortolanza S, Qian C, Kramer MG, et al. An oncolytic adenovirus controlled by a modified telomerase promoter is attenuated in telomerase-negative cells, but shows reduced activity in cancer cells. J Mol Med 2005; 83:736-47.

33 Takahashi M, Sato T, Sagawa T, et al. E1B-55K-deleted adenovirus expressing E1A-13S by AFP-enhancer/promoter is capable of highly specific replication in AFP-producing hepatocellular carcinoma and eradication of established tumor. Mol Ther 2002 ; 5:627-34.

$34 \mathrm{Kim}$ J, Lee B, Kim JS, et al. Antitumoral effects of recombinant adenovirus YKL-1001, conditionally replicating in alpha-fetoprotein-producing human liver cancer cells. Cancer Lett 2002; 180:23-32.

35 Bristol JA, Zhu M, Ji H, et al. In vitro and in vivo activities of an oncolytic adenoviral vector designed to express GM-CSF. Mol Ther 2003; 7:755-64.

36 Pei Z, Chu L, Zou W, et al. An oncolytic adenoviral vector of Smac increases antitumor activity of TRAIL against HCC in human cells and in mice. Hepatology 2004; 39: 1371-81. 
37 Zhao L, Gu J, Dong A, et al. Potent antitumor activity of oncolytic adenovirus expressing mda-7/IL-24 for colorectal cancer. Human Gene Therapy 2005;16: 845-58.

38 Liu X, Qiu S, Zou W, et al. Effective Gene-Virotherapy for Complete Eradication of Tumor Mediated by Combination of hTRAIL and Plasminogen K5. Mol Ther 2005; 11:531-41.

39 Prieto J, Qian C, Hernandez-Alcoceba R, et al. Gene therapy of liver diseases. Expert Opin Biol Ther 2004; 4:1073-91.

40 Clackson T. Regulated gene expression systems. Gene Ther 2000; 7:120-5.

41 Gossen M, Freundlieb S, Bender G, et al. Transcriptional activation by tetracyclines in mammalian cells. Science 1995; 268:1766-9.

42 Hoppe UC, Marban E, Johns DC. Adenovirus-mediated inducible gene expression in vivo by a hybrid ecdysone receptor. Mol Ther
2000; 1:159-64

43 Burcin MM, Schiedner G, Kochanek S, Tsai SY, O’Malley BW. Adenovirus-mediated regulable target gene expression in vivo. Proc Natl Acad Sci U S A 1999; 96:355-60.

44 Abruzzese RV, Godin D, Mehta V, et al. Ligand-dependent regulation of vascular endothelial growth factor and erythropoietin expression by a plasmid-based autoinducible GeneSwitch system. Mol Ther 2000; 2:276-87.

45 Wang L, Hernandez-Alcoceba R, Shankar V, et al. Prolonged and inducible transgene expression in the liver using gutless adenovirus: A potential therapy for liver cancer. Gastroenterology 2004; 126: 278-89.

46 Penuelas I, Mazzolini G, Boan JF, et al. Position emission tomography imaging of adenoviral-mediated transgene expression in liver cancer patients. Gastroenterology 2005; 128:1787-95. 